\title{
Reglas fiscales óptimas en un contexto de volatilidad del ciclo económico
}

Luis Morano Germani

\author{
Tesis de Maestría \\ Maestría en Economía \\ Universidad Nacional de La Plata
}

2019

Director de Tesis: Guillermo Vuletin Co-Director de Tesis: Jorge Puig 


\title{
Resumen
}

Este trabajo desarrolla un modelo teórico en el que se incorporan reglas fiscales directamente vinculadas con la sostenibilidad de la deuda: reglas de balance estructural, de presupuesto equilibrado y de límite a la deuda. La literatura sobre reglas fiscales aún no ha llegado a un consenso sobre qué tipo de reglas son óptimas en el contexto de economías pequeñas, sujetas a una alta volatilidad. El aporte de este trabajo es tratar las consecuencias de la implementación de dichas reglas en términos de bienestar en un marco común que permite hacer comparaciones. Las reglas de balance estructural maximizan bienestar y son preferibles a las reglas de deuda y de presupuesto equilibrado, donde estas últimas, además de distorsionar la suavización del consumo también maximizan la prociclicidad del gasto público. Estimaciones de panel para una muestra global para el período 1985-2015 sugieren que efectivamente las reglas de balance estructural disminuyen la volatilidad del consumo privado significativamente con respecto a los países que optan por reglas de presupuesto equlibrado.

\begin{abstract}
This paper develops a theoretical model that incorporates fiscal rules directly linked to debt sustainability: debt rules, and structural and balanced budget rules. The literature has not yet reached consensus on what kind of rules are optimal in the context of small economies, subject to high volatility. The contribution of this work is to deal with the consequences of adopting these rules in terms of well-being in a common framework that allows comparisons to be made. Structural balance rules maximize welfare and are preferable to debt rules and balanced budget rules, where the latter, in addition to distorting the smoothing of consumption (thus reducing welfare) also maximizes the procyclicality of public spending. Estimates of panel data for a global sample for the period 1985-2015 confirm that indeed the structural balance rules generate a significant decrease in the volatility of private consumption with respect to the countries that implement balanced budget rules.
\end{abstract}

Códigos JEL: E32, E60, E62, H50

reglas fiscales, bienestar, volatilidad, balance estructural 


\section{Índice}

1. Introducción 2

2. Revisión de la literatura $\quad 7$

3. Marco teórico 9

3.1. Modelo I: modelo base . . . . . . . . . . . . . . . . . . . . . . . 10

3.1.1. Problema del consumidor . . . . . . . . . . . . . . . . . . 10

3.1.2. Gobierno .................... 11

3.1.3. Restricciones agregadas . . . . . . . . . . . . . . . 12

3.1.4. Política fiscal óptima . . . . . . . . . . . . . . . . . . 13

3.1.5. El enfoque dual . . . . . . . . . . . . . . . . 14

3.1.6. Gasto alto del gobierno en $t=1$ : mecanismos de ajuste . . . 16

3.2. Incorporando reglas en un contexto de polítical fiscal óptima . . . . . 17

3.2.1. Modelo II: regla de balance estructural . . . . . . . . . . . . . 18

3.2.2. Modelo III: regla de presupuesto equilibrado . . . . . . . . . . 18

3.2.3. Modelo IV: regla de límite a la deuda . . . . . . . . . . . . . . 21

4. Aplicación empírica

5. Conclusiones 


\section{Introducción}

Durante las últimas tres décadas, en particular desde la fundación de la Unión Europea (UE) en 1992 con la firma del Tratado de Maastricht, las reglas fiscales han cobrado cada vez más relevancia en definir la sostenibilidad presupuestaria en el largo plazo.

En términos generales, las reglas fiscales pueden dividirse en dos categorías: reglas cuantitativas y reglas de procedimiento. ${ }^{1}$ Las primeras imponen algún tipo de restricción numérica de largo plazo en un agregado presupuestario, mientras que las segundas conciernen los procedimientos que aseguran que la política fiscal se lleve a cabo. Este trabajo se concentrará en el primer grupo de reglas, las cuantitativas.

De acuerdo al trabajo de Lledó et al. (2017), este tipo de reglas fiscales agregadas pueden agruparse en cinco categorías: ${ }^{2} 3$

I Reglas de límite al gasto: imponen un límite directo al gasto en términos nominales o reales. Si bien es relativamente fácil de comunicar y monitorear, no tiene una relación directa con la sostenibilidad de la deuda debido a que no restringe los ingresos. Un ejemplo reciente, en Brasil, es la implementación en el 2017 de una regla que limita el aumento del gasto público anual a la tasa de inflación registrada en el ejercicio anterior durante un plazo de dos décadas. ${ }^{4}$

II Reglas sobre los ingresos: imponen restricciones en la asignación de ingresos mayores que los esperados en épocas de bonanza. Tiene como objetivo aumentar la recaudación de ingresos y/o a prevenir una carga tributaria excesiva. Por sí sola podría resultar en una política fiscal procíclica, puesto que el establecer un límite mínimo que funcione como piso, no contribuye generalmente a la operación de los estabilizadores automáticos por el lado de los ingresos en una

$1 \quad$ Veáse Drazen (2013).

2 Basado en datos de Lledó et al. (2017). Esta base cuenta con datos de reglas fiscales agregadas en cinco categorías, para una muestra global para el período 1985-2015. Este trabajo es una actualización de las bases previamente publicadas por el Fondo Monetario Internacional (FMI).

3 Este trabajo se centra en el estudio de las reglas fiscales agregadas. No obstante, existen reglas fiscales de composición, las cuales imponen límites dentro de una categoría cubierta por una regla fiscal agregada. Por ejemplo, la regla de gasto de Perú, aprobada en el 2018, no sólo limita el crecimiento del gasto público general, sino también el del gasto corriente. Véase Izquierdo et al. (2018) y Ardanáz et al. (2019).

4 "El Congreso brasileño aprueba el Presupuesto para el 2017 con una nueva y polémica regla", Agencia EFE, 15 de diciembre del 2016. 
recesión; de igual manera, no tiene una relación directa con la sostenibilidad de la deuda. Sin embargo, puede controlar, de forma indirecta, el tamaño del gasto del gobierno. Esta regla, tal vez la menos común de las cinco, fue implementada por Australia entre 1985 y 1988; la ley comprometía a la autoridad económica a "no incrementar los ingresos como proporción del PIB over the life of the Parliament".

III Regla de límite a la deuda: impone límites en el monto de la deuda pública como porcentaje del PIB. Es relativamente fácil de comunicar y monitorear. Sin embargo, los niveles de deuda toman tiempo en ser impactados por las medidas presupuestarias y, por lo tanto, no proveen una guía clara en el corto plazo para los responsables de la política económica. Además, la política fiscal puede ser procíclica cuando la regla opera (como se explicará más adelante, en el margen, esta regla impide que la política fiscal funcione como amortiguador en tiempos malos). La regla de límite a la deuda de $60 \%$ del PIB adoptada por los países-miembro fundadores de la UE en 1992 es probablemente el ejemplo más conocido de éste tipo de regla.

IV Regla de presupuesto equilibrado: impone límites en el ingreso y gasto público. Al igual que las reglas precedentes, es fácil de comunicar y monitorear. Está en gran parte bajo el control de la autoridad económica, y provee una guía operacional clara. Si bien puede ayudar a asegurar la sostenibilidad de la deuda, no tiene funciones de estabilización económica. Desde 1991, Israel impone un objetivo nominal al balance fiscal. Los estados de EE. UU. son también otro ejemplo, de ente subnacional en este caso, que implementan reglas de presupuesto equilibrado.

V Regla de balance estructural: impone límites al balance fiscal ajustado cíclicamente. ${ }^{5}$ El balance fiscal estructural suele calcularse como el balance fiscal nominal ajustado por su componente cíclico, neto de medidas puntuales y temporales. Este componente se calcula como el producto de la brecha del producto (la diferencia entre el PIB actual y el potencial como porcentaje del

$\overline{5}$ El trabajo de Lledó et al. (2017) no distingue entre reglas de presupuesto equilibrado y balance estructural. Dicha diferenciación es posible al analizar las narrativas que presentan Lledó et al. (2017), país por país, y es un aporte de este trabajo. 
PIB potencial) y una elasticidad que refleja la reacción automática del balance del sector público a un cambio en la brecha del producto. Este equilibrio presupuestario ajustado cíclicamente, corresponde al que predominaría si la economía funcionara a niveles de tendencia. El ejemplo más destacado de regla de balance estructural es el de Chile, que implmenta dicha regla desde el $2001 .^{6}$

Es importante destacar que la adopción de este tipo de reglas por parte de los países no es excluyente. Como puede verse en la Figura 1, que abarca las reglas empleadas por una muestra global para el período 1985-2015 un $8 \%$ de la muestra cuenta en algún momento del tiempo con los cuatro tipos de regla en funcionamiento, este es el caso de Francia, por ejemplo. ${ }^{7}$

Figura 1: Distribución de reglas fiscales, por tipo. 1985-2015

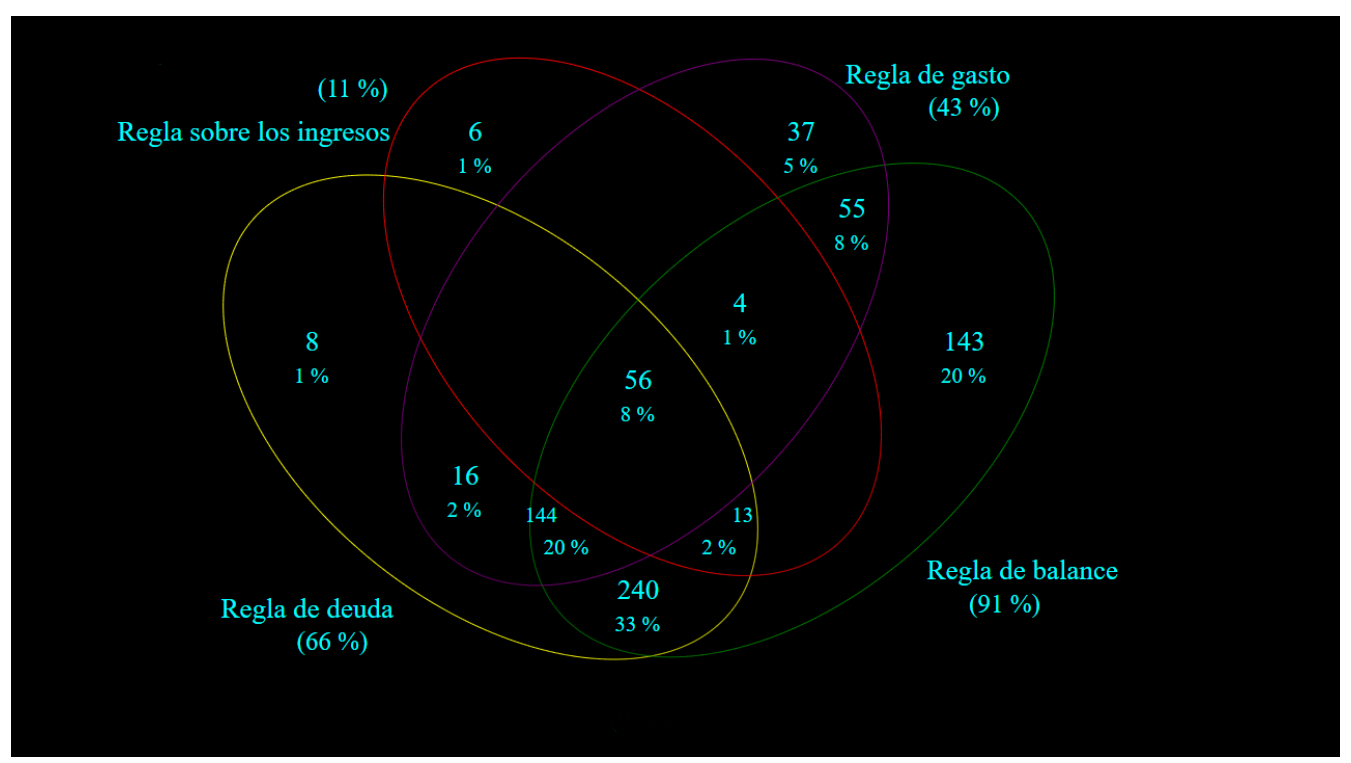

Fuente: elaboración propia basado en Lledó et al. (2017, FMI).

Desde mediados de la década de 1980 se ha visto un incremento sostenido en la adopción de reglas fiscales por parte de los países. Como puede observarse en la Figura 2, el crecimiento en la adopción de reglas fiscales ha sido sostenido. En particular, pueden observarse dos momentos en el tiempo muy claros en esta figura: un notable incremento en el número de reglas en 1992, año de implementación de

$6 \quad$ Véase, por ejemplo, Frankel (2011) para un estudio detallado del caso chileno.

7 Para que la representación gráfica del diagrama de Venn sea más clara, se agrupan en un mismo conjunto las reglas de balance equilibrado y estructural. 
los preceptos de Maastricht por parte de los países miembro de la UE, y una caída en el 2009, lo cual refleja el uso de cláusulas de escape por parte de algunos países luego de la Gran Recesión del 2008.

Figura 2: Evolución de reglas fiscales. 1985-2015

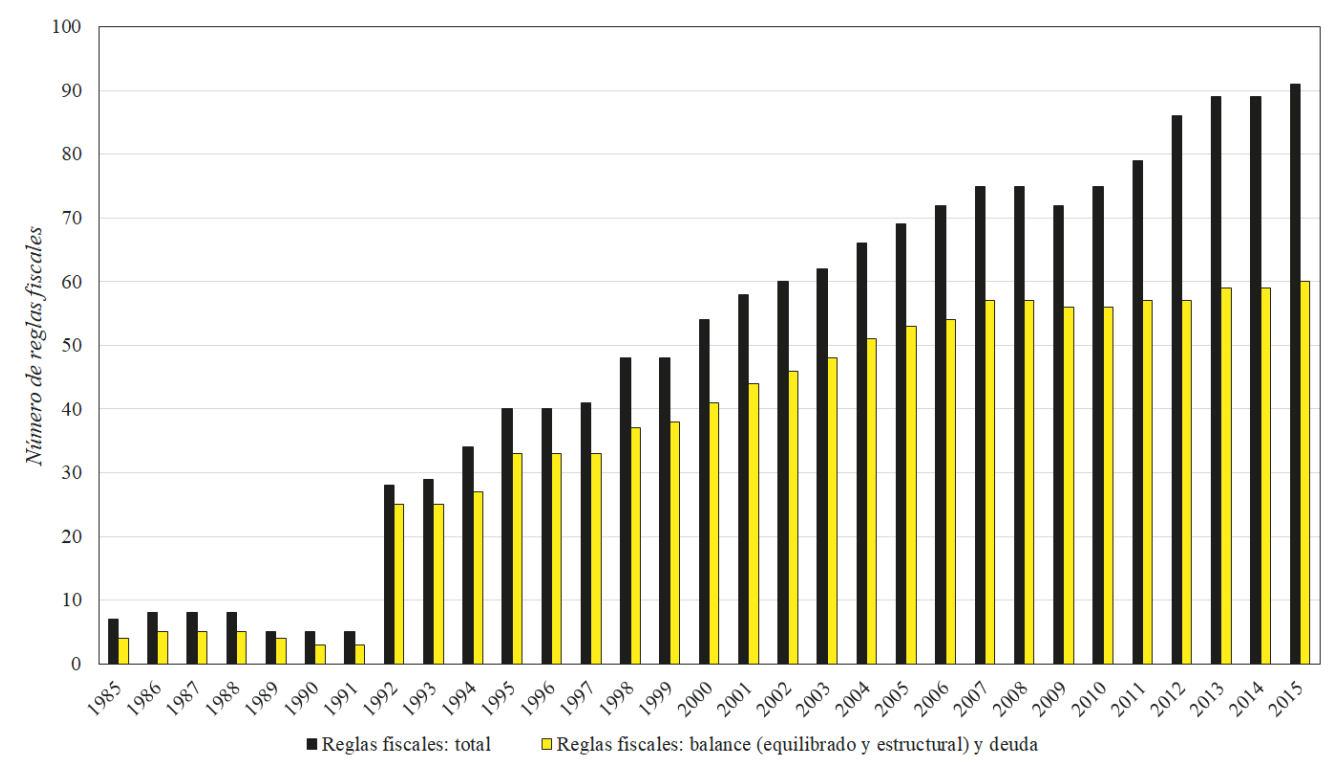

Fuente: elaboración propia basado en Lledó et al. (2017, FMI).

La adopción de dichas reglas responde a una preferencia explícita por parte de estos gobiernos por solucionar o mitigar algún fenómeno determinado. Las barras negras en la Figura 2 presentan el número total de reglas para un año dado. ${ }^{8}$ A su vez, las barras amarillas resumen los datos de un subgrupo de reglas: las de deuda, y las de presupuesto equilibrado y balance estructural. La razón por la cual este trabajo se enfocará en estas tres categorías de reglas se debe a que de las cinco mencionadas más arriba, son las únicas que tienen una vinculación directa con un objetivo de sostenibilidad de la deuda.

La Figura 3 presenta la participación relativa de cada una de estos tres tipos de regla para el total existente en cada año. Al concentrarnos en este subgrupo, observamos que a principios de la década de 1990 las reglas de balance estructural y de deuda no existían. El Tratado de Maastricht de 1992 explica la aparición de la barra oscura (la regla de un tope a la deuda de $60 \%$ del PIB previamente mencionada). No obstante, Maastricht no estipulaba ningún requerimiento en términos de reglas de balance

8 Es importante notar que aquí la observación es el par regla-país: un país con una regla de gasto y una regla de presupuesto equilibrado, como Israel, figurará dos veces. 
estructural: justamente Dinamarca, uno de los pocos países que había firmado el tratado con algunas excepciones, fue el primero en adoptar una regla de balance estructural, independiente de las reglas supranacionales impuestas por la UE. A lo largo de los años, como puede observarse, las barras rojas, correspondientes a las reglas de balance estructural, han incrementado su participación relativa.

Figura 3: Participación relativa de reglas vinculadas a sostenibilidad de la deuda, 1990-2015

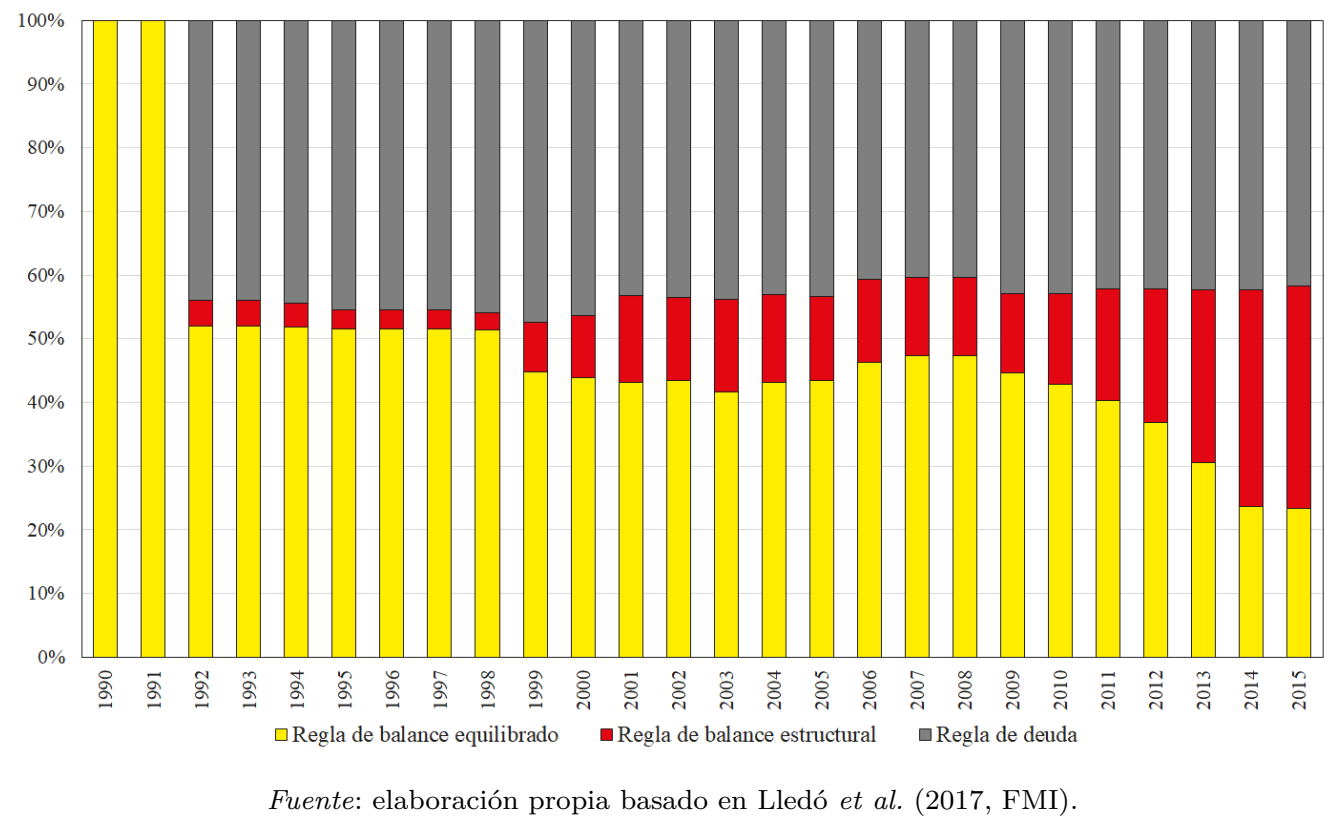

Este trabajo tiene como objetivo racionalizar la creciente adopción de reglas fiscales de balance estructural, por encima de las de presupuesto equilibrado y deuda. ¿Qué consecuencia teórica tiene la adopción de una regla u otra en términos de bienestar? ¿Qué sucede cuando evaluamos el desempeño de dichas reglas en un contexto de volatilidad económica, como es el caso de Argentina? El aporte de este trabajo se encuentra en la reducción del problema de elección de regla fiscal a un modelo teórico.

El trabajo concluye que las reglas fiscales de balance estructural son óptimas en términos de maximización de bienestar comparadas con las reglas de presupuesto equilibrado y deuda. Este resultado sugiere que el error en la elección de una regla fiscal se agrava a medida que aumenta la volatilidad del gasto público. La Sección 2 del trabajo resume la literatura vinculada a reglas fiscales, y desarrolla las distintas ramas que han tomado partido, bajo determinadas circunstancias, por alguno de estos tres tipos de regla. La Sección 3 presenta el modelo teórico y las principales 
conclusiones. En la Sección 4 se llevan a cabo estimaciones empíricas, que si bien no implican en modo alguno un vínculo causal, parecieran estar en línea con las conclusiones teóricas de la Sección 3. La Sección 5 concluye.

\section{Revisión de la literatura}

Como se menciona previamente, la implementación de reglas fiscales por parte de los países es aún un fenómeno reciente, aunque en ascenso. A su vez, la efectividad de las mismas está directamente relacionada con la solidez de las instituciones que las sostienen. En este contexto, es razonable esperar que los potenciales beneficios de la implementación de reglas fiscales no puedan observarse sino tras años de implementación e interacción con las instituciones que deben asegurar su cumplimiento. En este contexto, la literatura teórica sobre diseño óptimo de reglas fiscales discute los beneficios y costos de marcos fiscales basados en reglas, haciendo foco en el trade-off entre compromiso y flexibilidad (Amador et al. 2006; Halac y Yared, 2014 y Azzimonti et al. 2016). A su vez, Debrun y Jonung (2018) sugieren otra característica deseable para las reglas fiscales: simplicidad.

Por otra parte, como se muestra en el estudio de Eyraud et al. (2018), existe una gran variedad en el diseño de reglas fiscales en el mundo. Caselli y Reynaud (2019) muestran que cuando se controla por la endogeneidad entre reglas y resultados fiscales, un mejor diseño de reglas tiene un impacto significativo sobre el balance fiscal. Si bien las reglas fiscales comenzaron a aplicarse a mediados de la década de 1980, fue recién en 1992, con la adherencia de los doce países miembro de la UE al Tratado de Maastricht, que las mismas cobraron notoriedad. En ese contexto, Debrun et al. (2008) encuentran un efecto causal positivo de la implementación de reglas fiscales hacia un mejor manejo presupuestario: cuando el diseño no obstaculiza la función estabilización de las reglas, las mismas se asociacian con una menor prociclicidad. En un trabajo más reciente, Izquierdo et al. (2018) encuentran que la adopción de reglas fiscales está asociada a una menor prociclicidad y a una menor probabilidad de ocurrencia de una crisis de deuda. Por último, Asatryan et al. (2018) encuentran que la adopción constitucional de reglas de balance (sin distinguir si se trata de presupuesto equilibrado o balance estructural) reduce la probabilidad de ocurrencia 
de crisis de deuda soberana.

En cuanto a las reglas fiscales de balance estructural, Marcel (2013) repasa la experiencia chilena durante los primeros 10 años de su implementación, y destaca 10 lecciones que contribuirían en mejorar la regla en Chile o a su buena implementación en otros países de la región. Luego, en el marco de un modelo con dinero, sticky prices e impuestos distorsivos, Schimtt-Grohé y Uribe (2006), muestran que la política fiscal óptima, es una política fiscal pasiva (en el sentido de Leeper, 1991). ${ }^{9}$ Por último, Milesi-Ferretti (2004) advierte que cuando las reglas fiscales se imponen sobre variables "medibles", se crea un márgen para la "contabilidad creativa": la probabilidad de ocurrencia de "contabilidad creativa" depende del tamaño y del grado de transparencia del presupuesto.

En relación a las reglas de deuda, que como se explicará más adelante se ubican como un caso intermedio entre las reglas de balance estructural y presupuesto equilibrado en términos de bienestar, pocos trabajos señalan hacia la optimalidad de las mismas en contextos generales. Hatchondo et al. (2012) muestran que en el marco de un modelo de default, una regla de límite a la deuda surge como la política fiscal óptima: reduce la prociclicidad y la volatilidad del consumo. Este resultado no es sorprendente dado que surge de un modelo donde se permite que los países restructuren deuda voluntariamente de modo de apropiarse de las ganancias de capital implícitas en la reducción del spread que pagan. Nuevamente, el objetivo de este trabajo es evaluar la optimalidad de reglas fiscales en contextos más generales, y definitivamente no tan extremos como lo es una cesación de pagos.

Por último, con respecto a las reglas de presupuesto equilibrado (que es el tipo de regla sobre el que más se ha escrito) existe evidencia mixta sobre su optimalidad. El trabajo de Sorensen et al. (2001), para los estados de EE. UU. señala que las reglas de presupuesto equilibrado, por construcción, maximizan la prociclicidad. Este problema es uno de los tantos que surgen en estados federales, donde surge el common pool problem, donde los entes subnacionales no internalizan los costos que asumen los contribuyentes. ${ }^{10}$ Wyplosz (2012) realiza una revisión de la experiencia

$9 \quad$ En el contexto de un modelo estocástico, Leeper (1991) define una política pasiva como aquella está restringida por la optimización intertemporal de los agentes, por lo que debe generar suficientes recursos para equilibrar el balance intertemporal. Como se desarrollará más adelante, esa será la definición de regla fiscal de balance estructural a adoptarse en éste trabajo.

10 Sobre la prociclicidad de los estados brasileros y las provincias argentinas, véase Sturzenegger y Werneck (2006) y Gatti y Puig (2011). 
en la aplicación de reglas fiscales en estados federales, en el contexto inmediato a la crisis europea de deuda del 2009. Por otro lado, Alesina y Bayoumi (1996) argumentan que el efecto restrictivo de este tipo de reglas (que impide "buenas" políticas contracíclicas) en realidad compensa el desestabilizador voracity effect que ocurriría de no existir estas reglas. Siguiendo una lógica similar, Fatás y Mihov (2004) argumentan que las reglas de presupuesto equilibrado tienen dos efectos contrapuestos: (1) reducen la discresión de la política fiscal, y (2) reducen la respuesta de la autoridad fiscal ante shocks. Al igual que Alesina y Bayoumi, los autores argumentan que el primer efecto domina sobre el segundo, resignando una mayor volatilidad en favor de una menor discresionalidad de la política fiscal. Por último, Mendes y Pennings (2017) argumentan que en el contexto de economías pequeñas, exportadoras de materias primas, las reglas de presupuesto equilibrado son óptimas si los ingresos fiscales originados durante commodity booms se ahorran en un fondo soberano. El problema de este análisis es que descansa en el supuesto de una alta persistencia del shock sobre los ingresos, lo cual implícitamente asume la (casi) ausencia del ciclo económico. No obstante, la evidencia muestra que éste tipo de economías están sometidas a una alta volatilidad de sus ingresos fiscales, entonces, dado que los mismos están "atados" al ciclo económico, también lo estará el gasto, siendo esto la definición de la política fiscal procíclica.

La motiviación de este trabajo es enmarcar conceptualmente esta discusión en un modelo sencillo de una economía pequeña y abierta sujeta a una alta volatilidad, donde la adopción de reglas de presupuesto equilibrado es un error de política dado que la que maximiza el bienestar y minimiza la prociclicidad es la regla de balance estructural.

\section{Marco teórico}

A los efectos de este trabajo, se considerará una versión simplificada del modelo presentado en Stokey y Lucas (1983). ${ }^{11}$ El modelo a continuación cuenta con dos períodos en una economía pequeña y abierta, perfectamente integrada al resto del

11 Lucas y Stokey (1983) se concentran en la estructura y consistencia temporal de la política fiscal y monetaria en una economía sin capital. 
mundo tanto en mercados de bienes y de capital. ${ }^{12}$

\subsection{Modelo I: modelo base}

\subsubsection{Problema del consumidor}

Considere un modelo de dos períodos de una economía pequeña y abierta perfectamente integrada al resto del mundo

$$
W=\log \left(c_{1}\right)+\beta \log \left(c_{2}\right)
$$

donde $c_{1}$ denota el consumo en el período $t=1,2$ y $\beta<1$ es el factor de descuento. Los agentes comienzan su vida sin activos externos.

Las restricciones presupuestarias para los períodos 1 y 2 están dadas por, respectivamente,

$$
\begin{array}{r}
b_{1}=y_{1}-c_{1}\left(1+\theta_{1}\right), \\
0=y_{2}+(1+r) b_{1}-c_{2}\left(1+\theta_{2}\right),
\end{array}
$$

donde $b_{1}$ denota los activos externos netos al final del período $1, y_{t}$ es la dotación en el período $t(t=1,2), \theta_{1}$ es la tasa de un impuesto al consumo en el período $t$ y $r$ es la tasa de interés real internacional. Combinando las restricciones, obtenemos la restricción intertemporal del consumidor:

$$
y_{1}+\frac{y_{2}}{1+r}=c_{1}\left(1+\theta_{1}\right)+\frac{c_{2}\left(1+\theta_{2}\right)}{1+r}
$$

El consumidor elige $c_{1}$ y $c_{2}$ para maximizar la utilidad a lo largo de su vida, dada (1), sujeto a las restricciones intertemporales (3). En términos del Lagrangiano,

$$
\mathcal{L}=\log \left(c_{1}\right)+\beta \log \left(c_{2}\right)+\lambda\left\{y_{1}+\frac{y_{2}}{1+r}-c_{1}\left(1+\theta_{1}\right)+\frac{c_{2}\left(1+\theta_{2}\right)}{1+r}\right\}
$$

donde $\lambda$ is el multiplicador de Lagrange asociado a la restricción (3). Además de (3), las condiciones de primer orden están dadas por (suponiendo, como es habitual,

12 Véase Végh (2013) para un estudio exhaustivo de este tipo de modelos en contextos diversos. 
$\beta(1+r)=1)$

$$
\begin{aligned}
& \frac{1}{c_{1}}=\lambda\left(1+\theta_{1}\right), \\
& \frac{1}{c_{2}}=\lambda\left(1+\theta_{2}\right),
\end{aligned}
$$

Combinando estas dos condiciones de primer orden se obtiene la ecuación de Euler:

$$
\frac{c_{1}}{c_{2}}=\frac{1+\theta_{2}}{1+\theta_{1}}
$$

Esta condición de optimalidad se relaciona con la noción de distorsiones intertemporales. El precio del consumo enfrentado por los consumidores (incluyendo impuestos) es $1+\theta_{1}$ en el período 1 y $1+\theta_{2}$ en el período 2 . Si $\theta_{1}=\theta_{2}$, entonces no hay distorsión intertemporal y el consumo se suavizará a través del tiempo. Si $\theta_{1} \neq \theta_{2}$, existe una distorsión intertemporal y el perfil de consumo ya no se suavizará. Por lo tanto, si es factible, una política fiscal óptima implicará establecer una tasa impositiva constante sobre el consumo a lo largo del tiempo para evitar la introducción de una distorsión intertemporal.

\subsubsection{Gobierno}

El gobierno enfrenta un sendero exógeno de gasto público $\left\{g_{1}, g_{2}\right\}$ cuyo valor presente descontado debe ser financiado por la recaudación del impuesto al consumo. Además, el gobierno tiene acceso perfecto al mercado internacional de capitales y puede entonces tomar prestado o prestar a la tasa de interés internacional, $r$. Suponga que los activos externos netos iniciales son cero, entonces la restricción presupuestaria del gobierno está dada por

$$
\begin{gathered}
b_{1}^{g}=\theta_{1} c_{1}-g_{1}, \\
0=\theta_{2} c_{2}+(1+r) b_{1}^{g}-g_{2},
\end{gathered}
$$

donde $b_{1}^{g}$ denota los activos externos netos del gobierno. Sea el balance fiscal primario definido como el balance fiscal descontado del pago de intereses:

$$
B P_{t} \equiv \theta_{t} c_{t}-g_{t}
$$


$\mathrm{Si}$, por ejemplo, el gobierno incurre en un déficit primario en el período 1 (i.e., $\left.B P_{1}<0\right)$, deberá financiarlo pidiendo prestado. En el segundo período, necesitará generar un superávit primario para repagar la deuda más los intereses.

Combinando las restricciones presupuestarias del gobierno (5) y (6), obtenemos la restricción intertemporal: ${ }^{13}$

$$
\theta_{1} c_{1}+\frac{\theta_{2} c_{2}}{1+r}=g_{1}+\frac{g_{2}}{1+r}
$$

\subsubsection{Restricciones agregadas}

Combinando las restricciones del consumidor y del gobierno del período 1 - dadas por las ecuaciones (2) y (5), respectivamente - obtenemos la restricción agregada de la economía:

$$
b_{1}^{g}+b_{1}=y_{1}-\left(c_{1}+g_{1}\right)
$$

Por definición, la acumulación de activos externos netos de la economía es igual a la cuenta corriente. Entonces

$$
C C_{1}=y_{1}-\left(c_{1}+g_{1}\right)
$$

Es útil reescribir la cuenta corriente como la suma del ahorro privado y público. Sumando y restando $\theta_{1} c_{1}$ del lado derecho de la ecuación (9) obtenemos

$$
C C_{1}=\underbrace{y_{1}-\left(1+\theta_{1}\right) c_{1}}_{\text {Ahorro privado }}+\underbrace{\theta_{1} c_{1}-g_{1}}_{\text {Ahorro público }} .
$$

La suma del ahorro público y privado es el ahorro total de la economía. Por lo tanto, en un mundo sin inversión, la cuenta corriente es igual al ahorro. Esta forma de expresar la cuenta corriente apunta al vínculo entre los déficits primarios y de cuenta corriente. ${ }^{14}$ Es claro que, dado el ahorro privado, una caída en el ahorro público incrementará el déficit de cuenta corriente. No obstante, en equilibrio, esta relación entre el déficit fiscal y la cuenta corriente no necesariamente se mantiene porque el ahorro privado puede reaccionar también a cualquier shock que afecte al

13 Para asegurar un consumo positivo, se asume que el valor presente descontado del gasto público es menor al valor presente descontado del producto; esto es, $g_{1}+g_{2} / 1+r<y_{1}+y_{2} / 1+r$ (la condición de transversalidad).

14 Esto no son otra cosa que los "déficits gemelos". 
ahorro público.

Combinando las restricciones intertemporales de los consumidores y del gobierno (dadas por las ecuaciones (3) y (8), respectivamente), obtenemos la restricción de recursos de la economía:

$$
y_{1}+\frac{y_{2}}{1+r}=c_{1}+g_{1}+\frac{c_{2}+g_{2}}{1+r} .
$$

Notar que las restricciones agregadas no dependen de las tasas del impuesto al consumo porque los pagos de impuestos constituyen una transferencia entre consumidores y el gobierno.

\subsubsection{Política fiscal óptima}

El gobierno debe financiar el sendero de gasto público estableciendo las tasas del impuesto $\theta_{1}$ y $\theta_{2}$. ¿Cuáles son los valores óptimos de $\theta_{1}$ y $\theta_{2}$ ? La noción de optimalidad presupone que se cuenta con una función objetivo bien definida. En un marco de optimización, la función objetivo natural del gobierno es la utilidad de por vida del consumidor. Por lo tanto, la política fiscal óptima se define como aquellos valores de $\theta_{1}$ y $\theta_{2}$ que maximizan el bienestar del consumidor sujeto a la restricción de que el valor presente descontado de los ingresos fiscales sea suficiente para financiar el valor presente descontado del gasto público.

Metodológicamente, hay dos formas de encontrar los valores óptimos de $\theta_{1}$ y $\theta_{2}$. El primero, el enfoque más común en las finanzas públicas, es el enfoque dual. En este enfoque, primero resolvemos el problema del consumidor con $c_{1}$ y $c_{2}$ como funciones de $\theta_{1}$ y $\theta_{2}$. Luego, sustituimos en la función de utilidad para obtener la función de utilidad indirecta (es decir, la utilidad como una función de $\theta_{1}$ y $\theta_{2}$ ). El gobierno elige $\theta_{1}$ y $\theta_{2}$ para maximizar esta función de utilidad indirecta sujeta a su propia restricción intertemporal. El problema con el enfoque dual es que no funcionará en problemas más complicados donde puede ser difícil obtener la función de utilidad indirecta. El segundo enfoque es el enfoque primal y es, por mucho, el enfoque más común en macroeconomía. Implica resolver el problema de Ramsey. El planificador de Ramsey es un planificador restringido en el sentido de que elige cantidades (como lo haría un planificador central) pero sujeto a la restricción de que la asignación elegida puede implementarse como un equilibrio competitivo (tales restricciones se 
conocen como condiciones de implementabilidad) .

\subsubsection{El enfoque dual}

El primer paso en el enfoque dual de la tributación óptima es resolver las funciones de demanda del consumidor. Sustituyendo la ecuación de Euler (4) en la restricción intertemporal (3), obtenemos

$$
\begin{aligned}
& c_{1}=\tilde{c}_{1}\left(\theta_{1}, Y\right) \equiv \frac{1}{1+\theta_{1}}\left(\frac{1+r}{2+r}\right) Y \\
& c_{2}=\tilde{c}_{2}\left(\theta_{2}, Y\right) \equiv \frac{1}{1+\theta_{2}}\left(\frac{1+r}{2+r}\right) Y .
\end{aligned}
$$

donde $\tilde{c}_{1}($.$) y \tilde{c}_{2}($.$) denotan funciones e$

$$
Y \equiv y_{1}+\frac{y_{2}}{1+r}
$$

es el valor presente descontado del producto. La demanda de los consumidores depende entonces positivamente de $Y$ y negativamente de la tasa del impuesto.

Sustituyendo las demandas de los consumidores (12) y (13) en la utilidad de por vida del consumidor (1), obtenemos el bienestar del consumidor como función de las tasas impostivas $\theta_{1}$ y $\theta_{2}$ :

$$
W\left(\theta_{1}, \theta_{2}\right)=\log \left(\tilde{c}_{1}\left(\theta_{1}, Y\right)\right)+\beta \log \left(\tilde{c}_{2}\left(\theta_{2}, Y\right)\right)
$$

El gobierno elige $\theta_{1}$ y $\theta_{2}$ para maximizar la función de utilidad indirecta, dada por (14), sujeta a la restricción intertemporal del gobierno (8). El Lagrangiano entonces es

$$
\mathcal{L}=\log \left(\tilde{c}_{1}\left(\theta_{1}, Y\right)\right)+\beta \log \left(\tilde{c}_{2}\left(\theta_{2}, Y\right)\right)+\Psi\left\{\theta_{1} \tilde{c}_{1}\left(\theta_{1}, Y\right)+\frac{\theta_{2} \tilde{c}_{2}\left(\theta_{2}, Y\right)}{1+r}-g_{1}-\frac{g_{2}}{1+r}\right\}
$$

donde $\Psi$ es el multiplicador asociado con la restricción (8).

Bajo el supuesto de que $\beta(1+r)=1$, las condiciones de primer orden con respecto 
a $\theta_{1}$ y $\theta_{2}$ están dadas por, respectivamente,

$$
\begin{gathered}
\frac{1}{\tilde{c}_{1}\left(\theta_{1}, Y\right)} \frac{\partial \tilde{c}_{1}\left(\theta_{1}, Y\right)}{\partial \theta_{1}}=-\Psi \frac{\partial\left(\theta_{1} \tilde{c}_{1}\left(\theta_{1}, Y\right)\right)}{\partial \theta_{1}} \\
\frac{1}{\tilde{c}_{2}\left(\theta_{2}, Y\right)} \frac{\partial \tilde{c}_{2}\left(\theta_{2}, Y\right)}{\partial \theta_{2}}=-\Psi \frac{\partial\left(\theta_{2} \tilde{c}_{2}\left(\theta_{2}, Y\right)\right)}{\partial \theta_{2}}
\end{gathered}
$$

Pero notar que de (12) y (13) se deduce que

$$
\begin{aligned}
& \frac{\partial\left(\theta_{1} \tilde{c}_{1}\left(\theta_{1}, Y\right)\right)}{\partial \theta_{1}}=-\frac{\partial \tilde{c}_{1}\left(\theta_{1}, Y\right)}{\partial \theta_{1}} \\
& \frac{\partial\left(\theta_{2} \tilde{c}_{2}\left(\theta_{2}, Y\right)\right)}{\partial \theta_{2}}=-\frac{\partial \tilde{c}_{2}\left(\theta_{2}, Y\right)}{\partial \theta_{2}}
\end{aligned}
$$

Tomando en cuenta las últimas dos ecuaciones, podemos reducir las condiciones de primer orden (15) y (16) a

$$
\begin{aligned}
& \frac{1}{\tilde{c}_{1}\left(\theta_{1}, Y\right)}=\Psi \\
& \frac{1}{\tilde{c}_{2}\left(\theta_{2}, Y\right)}=\Psi
\end{aligned}
$$

De (17) y (18) se distingue que $\tilde{c}_{1}\left(\theta_{1}, Y\right)=\tilde{c}_{2}\left(\theta_{2}, Y\right)$ y por lo tanto de (12) y (13) que $\theta_{1}=\theta_{2}=\theta$. Es entonces óptimo para el gobierno mantener la tasa impositiva constante a través del tiempo de forma de no introducir ninguna de distorsión intertemporal, asegurando la suavización del consumo intertemporal.

Para obtener una forma reducida del consumo, sustituimos $c_{1}=c_{2}=c$ en la restricción de recursos de la economía (11) para obtener

$$
c=\left(\frac{1+r}{2+r}\right)(Y-G)
$$

donde $G\left(\equiv g_{1}+g_{2} /(1+r)\right)$ es el valor presente descontado del gasto público. Sustituyendo (19) en la restricción intertemporal del gobierno (8) se obtiene una expresión para la tasa constante del impuesto al consumo:

$$
\theta=\frac{G}{Y-G}
$$

La tasa impositiva óptima es entonces una función creciente del valor presente descontado del gasto público y no depende del sendero particular del gato público 
$\left(g_{1}, g_{2}\right)$. Intuitivamente, el gobierno puede suavizar tasas a través del tiempo tomando prestado cuando el gasto es alto y repagando cuando es bajo.

Una observación importante es que la política fiscal óptima replica el escenario de impuesto de suma fija. Esto es, el hecho de que la tasa del impuesto sea constante a través del tiempo implica que existe distorsión intertemporal, y el hecho de que el modelo no contemple el ocio implica que no hay distorsión intratemporal. Así entonces se replica el caso de impuesto de suma fija en el cual la restricción intertemporal del consumidor estaría dada por

$$
y_{1}+\frac{y_{2}}{1+r}=c_{1}+\frac{c_{2}}{1+r}-T
$$

donde $T$ denota el impuesto de suma fija. En este caso, el consumidor tampoco enfrentaría distorsiones intertemporales.

\subsubsection{Gasto alto del gobierno en $t=1$ : mecanismos de ajuste}

Suponga un escenario en el cual el gasto del gobierno en el período 1 es mayor al del período 2 (esto es, $g_{1}>g_{2}$ ) y suponga a su vez que la dotación de ingreso $y_{1}=y_{2}=y$ es constante a través del tiempo para abstraernos del motivo ahorro.

Primero chequeamos que, como es esperable, el ahorro privado será cero. Dado que los consumidores no enfrentan una distorsión intertemporal (esto es, la tasa del impuesto es constante a través del tiempo) y el sendero de la dotación es constante a través del tiempo, no hay motivo para ahorrar o desahorrar. Usando (19) y (20), podemos computar el nivel constante de gasto privado:

$$
c(1+\theta)=\frac{1+r}{2+r} Y
$$

Recordar que el ahorro privado está dado por

$$
S_{1}=y_{1}-c_{1}\left(1+\theta_{1}\right)
$$

Dado $y_{1}=y$ y $c_{1}\left(1+\theta_{1}\right)=c(1+\theta)$, sustituyendo (22) en (23) prueba que $S_{1}=0$. En cuanto al balance primario (ahorro público), usando (19) y (20) se obtiene el 
nivel constante de ingresos tributarios

$$
\theta c=\left(\frac{1+r}{2+r}\right) G
$$

Sustituyendo esta ecuación en la definición de balance fiscal primario - dada por (7) - y recordando que $G \equiv g_{1}+g_{2} /(1+r)$,

$$
\begin{array}{r}
B P_{1}=\frac{g_{2}-g_{1}}{2+r}<0, \\
B P_{2}=\frac{1+r}{2+r}\left(g_{1}-g_{2}\right)>0 .
\end{array}
$$

Dado que $g_{1}>g_{2}$, el gobierno incurrirá en un déficit fiscal primario en el primer período (i.e., $\left.B P_{1}<0\right)$ que es financiado con crédito del exterior. En el segundo período, el gobierno genera un superávit primario (i.e., $B P_{2}>0$ ) que le permite repagar la deuda más los intereses. Se deduce de (10) que la economía tendrá un déficit de cuenta corriente en el período 1. Es esta entonces una situación en la cual déficits gemelos (déficit primario y de cuenta corriente) surgen como respuesta óptima a un gasto temporalmente alto del gobierno. ${ }^{15}$

Notar que debiera ser intuitivamente claro que el resultado de suavización del consumo no depende del supuesto de preferencias logarítmicas. Incluso bajo una especificación más general de las preferencias, no hay razón para el gobierno para imponer una distorsión intertemporal costosa si puede evitarse.

\subsection{Incorporando reglas en un contexto de polítical fiscal óptima}

El modelo desarrollado en la sección anterior provee una referencia teórica sobre la optimalidad de la política fiscal, es decir, que maximiza el bienestar. En principio, sería deseable que cualquier modificación que se haga a dicho marco (para incorporar una regla fiscal, por ejemplo) mantenga el mismo nivel de bienestar que el que alcanza la economía en el Modelo 1. Las siguientes subsecciones incorporarán al modelo base una regla fiscal de balance estructural, una regla de presupuesto

\footnotetext{
15 Si por el contrario, $y_{1}>y_{2}$ y el sector privado hubiere ahorrado en el primer período, podría observarse un equilibrio con déficit fiscal y superávit de cuenta corriente.
} 
equilibrado y una regla de deuda, respectivamente, y evaluarán los resultados en términos de bienestar.

\subsubsection{Modelo II: regla de balance estructural}

Como se explicó anteriormente, una regla fiscal de balance estructural, al menos en su estado más puro, estima los ingresos del gobierno ajustados por el ciclo económico y, consecuentemente, autoriza un gasto público consistente con dichos ingresos. ${ }^{16}$ Lo que hace este tipo de regla entonces, es fijar un objetivo para el balance estructural para algún punto en el tiempo. Esto es, el gasto permanente del gobierno debiera igualar su ingreso permanente. Notar que en términos del modelo presentado en la sección anterior, dicha condición está implícita en la ecuación (7): empleando la definición de balance primario, podemos reescribir esta expresión como

$$
B P_{1}+\frac{B P_{2}}{1+r}=0
$$

Esto quiere decir que el modelo base desarrollado en la sección anterior, el Modelo I, tiene implícita una regla de balance estructural. Trivialmente, los resultados en términos de bienestar que pueden obtenerse del primer modelo pueden ser perfectamente replicados por el segundo porque efectivamente son idénticos: impuestos no distorsivos a través del tiempo $\theta_{1}=\theta_{2}$ y suavización intertemporal del consumo $c_{1}=c_{2}$. En términos de bienestar: $W_{I}=W_{I I}$.

\subsubsection{Modelo III: regla de presupuesto equilibrado}

Una regla de presupuesto equilibrado esencialmente impone límites en el ingreso y gasto públicos de forma conjunta. Este tipo de reglas suelen aplicarse sobre el balance primario, en oposición al balance financiero, dado que en éste último intervienen los intereses de la deuda, variable que no está bajo control de las autoridades económicas. Como se ha documentado en varios trabajos, una consecuencia directa de la implementación de este tipo de reglas es un elevado grado de procilicalidad del

$\overline{16}$ En el caso de países cuyos ingresos públicos dependen en gran medida de los precios internacionales de las materias primas (en particular, del precio de recursos naturales no renovables), la estimación del ingreso ajustado por el ciclo económico debe contemplar esta potencial fuente de volatilidad de los ingresos. 
gasto público, en particular en entes subnacionales, como son los estados de EE.UU. La Figura 4 muestra la correlación de los componentes cíclicos del gasto público y del producto para los estados de Estados Unidos. Independientemente de lo que ocurre a nivel federal, donde el país es marcadamente contracíclico, los estados exhiben un gasto público casi exclusivamente procíclico.

La razón por la cual esta regla maximiza la prociclidad, se debe a que, todo lo demás Figura 4: Correlación entre componentes cíclicos de producto y gasto real, por estado, 1963-2005

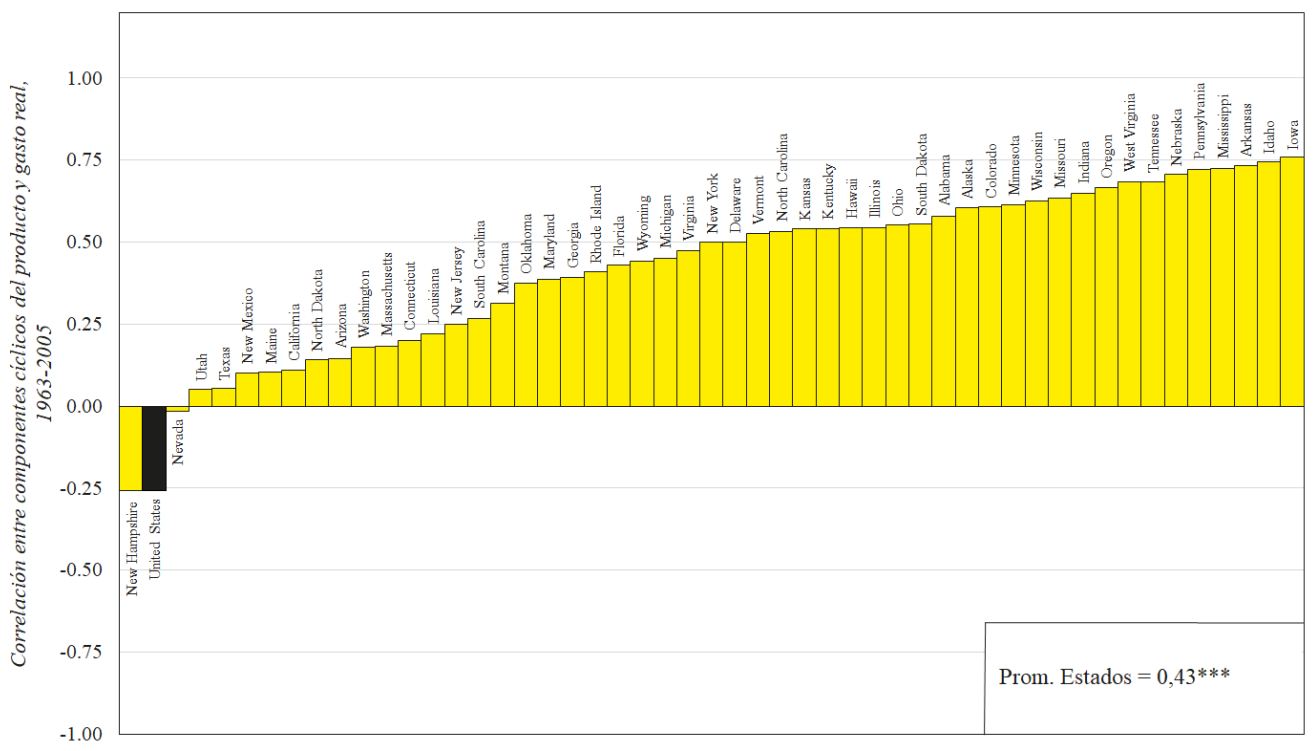

Fuente: elaboración propia basado en U.S. Census Bureau.

constante, los ingresos fiscales se "atan" a las fluctuaciones del ciclo económico, por lo que para cumplir con la regla y balancear ingresos y gastos, será necesario gastar más en buenos tiempos, y viceversa. ${ }^{17}$

Supongamos ahora que el mismo gobierno de los modelos I y II, opta por una regla de presupuesto equilibrado. En particular, opta por una regla donde, en cada período, los ingreso deben financiar la totalidad de los gastos. A los efectos prácticos del modelo esto implica:

$$
b_{1}^{g}=0
$$

Intuitivamente, este supuesto tiene como implicancia directa la eliminación del canal

17 Véase la Figura A1 en el Apéndice, donde se muestra que la correlación de los componentes cíclicos de los ingresos tributarios y el producto real, tienen una correlación positiva y estadísticamente significativa, tanto para países emergentes como para países avanzados. 
de financiamiento intertemporal para el gobierno: ya no existe la restricción intertemporal determinada por la ecuación (8), sino que ahora habrá $t=2$ restricciones de balance:

$$
\begin{aligned}
& g_{1}=\theta_{1} c_{1}, \\
& g_{2}=\theta_{2} c_{2},
\end{aligned}
$$

Notar a su vez, que esto implica que la ecuación (10) ahora estará dada por $C C_{1} \equiv$ Ahorro privado dado que el gobierno ya no tiene la oportunidad de financiarse intertemporalmente incurriendo en un déficit primario.

Dado que la decisión óptima del consumidor permanece inalterada (no así su consumo efectivo, como quedará claro más adelante), el nuevo problema de optimización a resolver por parte del planificador pasa a ser:

$$
\mathcal{L}=\log \left(\tilde{c}_{1}\left(\theta_{1}, Y\right)\right)+\beta \log \left(\tilde{c}_{2}\left(\theta_{2}, Y\right)\right)+\Psi_{1}\left\{\theta_{1} \tilde{c}_{1}\left(\theta_{1}, Y\right)-g_{1}\right\}+\Psi_{2}\left\{\theta_{2} \tilde{c}_{2}\left(\theta_{2}, Y\right)-g_{2}\right\}
$$

Las condiciones de primer orden con respecto a $\theta_{1}, \theta_{2}, \Psi_{1}$ y $\Psi_{2}$ están dadas por:

$$
\begin{gathered}
\frac{1}{\tilde{c}_{1}\left(\theta_{1}, Y\right)} \frac{\partial \tilde{c}_{1}\left(\theta_{1}, Y\right)}{\partial \theta_{1}}=-\Psi_{1} \frac{\partial\left(\theta_{1} \tilde{c}_{1}\left(\theta_{1}, Y\right)\right)}{\partial \theta_{1}} \\
\frac{1}{\tilde{c}_{2}\left(\theta_{2}, Y\right)} \frac{\partial \tilde{c}_{2}\left(\theta_{2}, Y\right)}{\partial \theta_{2}}=-\Psi_{2} \frac{\partial\left(\theta_{2} \tilde{c}_{2}\left(\theta_{2}, Y\right)\right)}{\partial \theta_{2}} \\
\theta_{1} \tilde{c}_{1}\left(\theta_{1}, Y\right)=g_{1} \\
\theta_{2} \tilde{c}_{2}\left(\theta_{2}, Y\right)=g_{2}
\end{gathered}
$$

Notar que haciendo uso de (12) y (13), y empleando (27) y (28), las condiciones de primer orden (25) y (26) son:

$$
\begin{gathered}
\frac{1}{\tilde{c}_{1}\left(\theta_{1}, Y\right)}=\Psi_{1} \\
\frac{1}{\tilde{c}_{2}\left(\theta_{2}, Y\right)}=\Psi_{2} .
\end{gathered}
$$

Y las tasas del impuesto al consumo son:

$$
\theta_{1}=\frac{g_{1}}{\tilde{c}_{1}\left(\theta_{1}, Y\right)}
$$




$$
\theta_{2}=\frac{g_{2}}{\tilde{c}_{2}\left(\theta_{2}, Y\right)}
$$

Puede intuirse ya que podrá replicarse el equilibrio con suavización del consumo si y sólo si $g_{1}=g_{2}$. Sustituyendo las nuevas tasas del impuesto en el problema de optimización del consumidor se obtienen las nuevas demandas para los períodos 1 y 2:

$$
\begin{aligned}
c_{1} & =\frac{1+r}{2+r} Y-g_{1} \\
c_{2} & =\frac{1+r}{2+r} Y-g_{2}
\end{aligned}
$$

Efectivamente, los consumos del sector privado en los períodos 1 y 2 dependen del gasto corriente en cada período. Esto significa que ante cualquier sendero de gasto que presente algún grado de volatilidad, se perderá el resultado de maximización de bienestar que implica la suavización del consumo. Puede observarse a su vez, que cuanto mayor la diferencia entre $g_{1}$ y $g_{2}$, mayor será la distorsión en el sendero de consumo, por lo que mayor será la pérdida en el bienestar. Así, $W_{I I I}<W_{I I}=W_{I}$

\subsubsection{Modelo IV: regla de límite a la deuda}

Notar que a los efectos prácticos del modelo de dos períodos aquí presentado, un límite a la deuda $\bar{b}$ puede interpretarse como un caso intermedio entre la regla de balance estructural y la de presupuesto equilibrado.

Mientras $b_{1}^{g}<\bar{b}$ el sector público podrá financiarse intertemporalmente, continuando en el mundo del Modelo II y maximizará $W$ ya que $\theta_{1}=\theta_{2}$ es óptimo siempre.

Cuando $b_{1}^{g}=\bar{b}$ el sector público pasará a estar en autarquía financiera, por lo que se verá obligado a elegir $\theta_{1} \neq \theta_{2}$, rompiendo así con la suavización del consumo y disminuyendo $W$ con respecto al óptimo. ${ }^{18}$

Entonces, el caso de una regla de límite a la deuda puede entenderse como un caso intermedio de los Modelos 2 y 3 . El resultado en términos de bienestar estará más cerca de uno u otro dependiendo de qué tan operativo sea el límite $\bar{b}$ :

- si el gobierno alcanza rápidamente $\bar{b}$, entonces $W_{I V}$ estará más cerca de $W_{I I I}$,

- a la inversa, cuanto más tarde el gobierno en alcanzar $\bar{b}$, el resultado de bien-

18 Notar que para la regla de presupuesto equilibrado $\bar{b}=0$, por lo que el financiamiento intertemporal se corta por completo. 
estar $W_{I V}$ se ubicará más próximo al de $W_{I I} \cdot{ }^{19}$

En cualquier caso, $W_{I I I}<W_{I V}<W_{I I}=W_{I}$.

\section{Aplicación empírica}

En la siguiente sección, se lleva a cabo una aplicación empírica donde se pone a prueba la principal predicción del modelo, la maximización del bienestar por parte de las reglas de balance estructural por sobre las de presupuesto equilibrado. A su vez, se evalúa una implicancia secundaria, que consiste en que las reglas de balance estructural reducen la prociclicidad del gasto público. Para estos fines se emplean datos de la mencionada base de Lledó et al. (2017) y del World Economic Outlook (WEO) del FMI de abril del 2019.

Dado que en el modelo la maximización del bienestar viene dada por la suavización del consumo, se utilizará para las estimaciones la volatilidad de la tasa de crecimiento del consumo privado como proxy de bienestar. Como es habitual en la literatura (por ejemplo, Ramey y Ramey, 1995) la volatilidad está medida por la desviación estándar de la tasa de crecimiento. ${ }^{20}$ Por otra parte, la prociclicidad del gasto público está medida como la correlación entre los componentes cíclicos del producto y gasto real. ${ }^{21}$ El Cuadro A1, en el Apéndice resume las estadísticas descriptivas de estas variables. Notar que los datos ya sugieren una menor volatilidad del consumo privado y menor prociclicidad de aquellos países que optan por una regla de balance estructural.

La manera en que se estima la relación entre reglas fiscales, por un lado, y volatilidad del consumo privado y prociclicidad del gasto público, por el otro, es mediante la siguiente especificación empírica:

$$
y_{i, t}=\alpha_{i}+\beta_{1} F R_{i, t}+\beta_{2} X_{i, t}+\beta_{3} \text { tendencia }+\beta_{4} \text { tendencia }{ }^{2}+\mu_{i, t}
$$

donde $y_{i, t}$ representa la variable dependiente de interés relevante en cada caso (volatilidad del consumo privado en el Cuadro 1 y prociclicidad del gasto público en el

\footnotetext{
19 La naturaleza de la regla de deuda es distinta a las de balance en el sentido de que se aplica a un stock, no a un flujo. Una regla de límite a la deuda cobraría más sentido en un modelo con infinitos períodos, que permitiría observar movimientos en dicho stock de deuda.

20 Véase Ramey y Ramey (1995).

21 Véase Frankel, Végh y Vuletin (2013).
} 
Cuadro 2) y $F R_{i, t}$ es una variable que toma valores entre cero y uno, capturando, como se detalla más abajo, la intensidad en la implementación de la regla fiscal. $X_{i, t}$ denota un vector de variables de control (crecimiento de la población y ratio de deuda sobre PIB) y $\alpha_{i}$ incorpora efectos fijos por país. Adicionalmente, en las estimaciones aquí presentadas se utiliza una tendencia lineal y una tendencia cuadrática al igual que en Owyang, Ramey, y Zubairy (2013) y Riera-Crichton, Végh y Vuletin (2015). Cada observación está calculada como una ventana móvil de 10 años, el período de estimación es 1985-2015 y la muestra de países incluidos en las estimaciones se detalla en el Apéndice.

La variable $F R_{i, t}$ se define de tres formas distintas: ${ }^{22}$

- Tiempo con regla fiscal: porporción de años durante la cual el país $i$ adoptó alguna de las tres reglas vinculadas a la sostenibilidad de la deuda.

- Tiempo con BBR (por su sigla en inglés, balanced budget rule): porporción de años durante la cual el país $i$ adoptó una regla de presupuesto equilibrado.

- Tiempo con SBR (por su sigla en inglés, structural balance rule): porporción de años durante la cual el país $i$ adoptó una regla de balance estructural.

El Cuadro 1 resume los resultados de las estimaciones con respecto a la volatilidad del consumo privado. Puede observarse en la columna (1) que la adopción de alguna de las tres reglas vinculadas a la sostenibilidad de la deuda tiene un efecto negativo pero no significativo sobre la volatilidad del consumo privado. No obstante, cuando se incorporan en la regresión las dos reglas "extremas" de los modelos II y III por separado en la columna (2), observamos que la implementación de una regla de balance estructural tiene un efecto, ahora sí, estadísticamente sifnificativo en la reducción de la volatilidad del consumo privado. De hecho, al analizar los efectos de estas dos reglas por separado, columnas (3) y (4), puede observarse que la regla de balance estructural disminuye significativamente la volatilidad del consumo, mientras que la de presupuesto equilibrado tiene un efecto positivo aunque no significativo.

22 Notar que, como consecuencia de haber definido la variable en una ventana móvil, la misma se interpreta como la proporción de años dentro de la ventana durante la cual el país $i$ adoptó una regla determinada 
Cuadro 1: Regresiones de panel con efectos fijos sobre la volatilidad del consumo privado con ventana móvil de 10 años

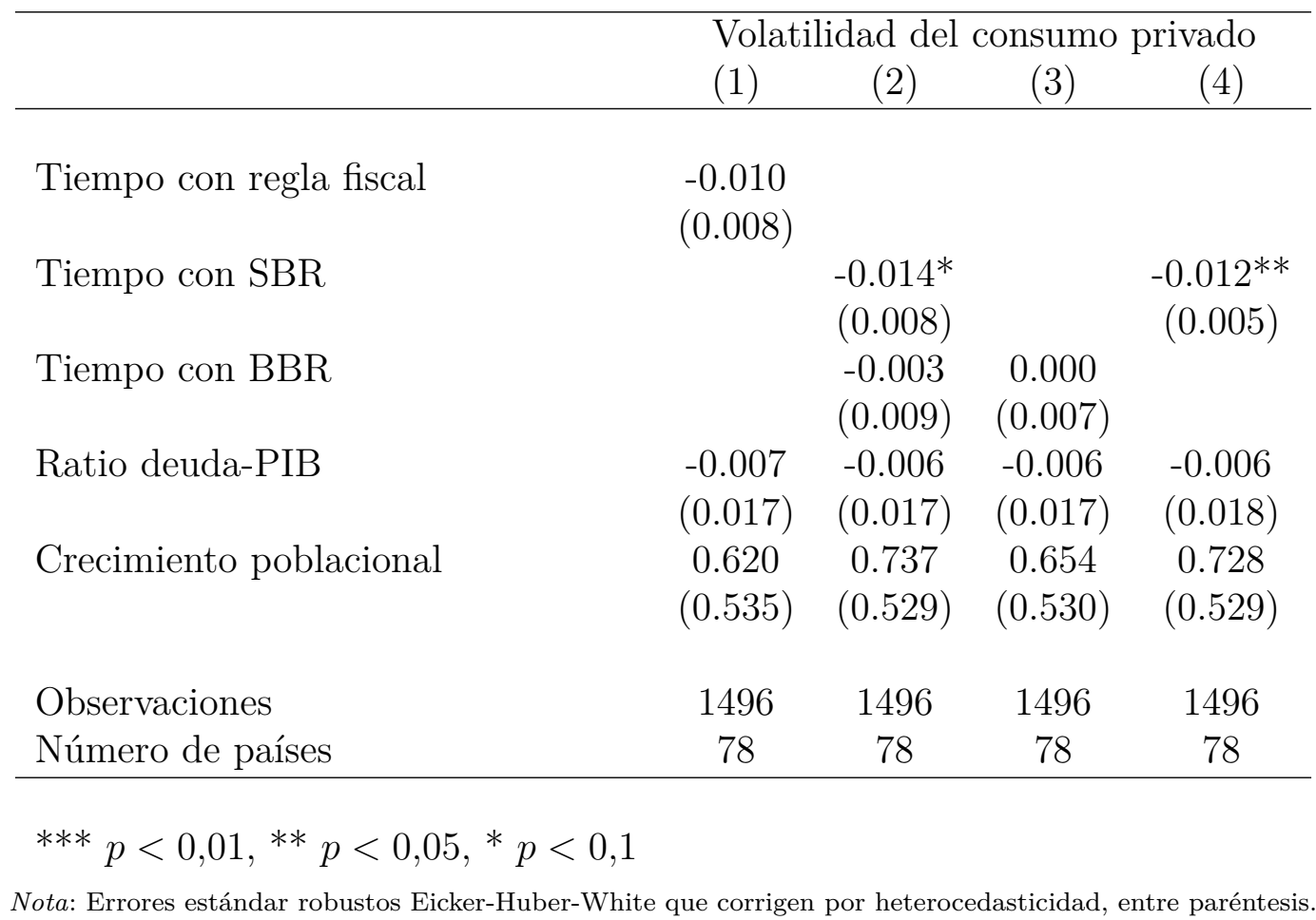

El Cuadro 2 resume los resultados de las estimaciones con respecto a la prociclicidad del gasto público. Al igual que en el Cuadro 1, en la columna (1) se presenta el coeficiente para la adopción de alguna de las tres reglas vinculadas a la sostenibilidad de la deuda. El coeficiente es positivo, aunque no significativo. No obstante, indagando un poco más dentro del tipo de regla fiscal que se aplica, puede observarse en la columna (2) que la regla de balance estructural disminuye significativamente la prociclicidad. Además, cuando estas reglas se analizan por separado, columnas (3) y (4), observamos que la regla de presupuesto equilibrado aumenta la prociclidad, mientras que la regla de balance estructural nuevamente la reduce. Este resultado es consistente con la literatura y las implicancias teóricas de la implementación de una y otra regla.

Las estimaciones son robustas a cambios en la ventana móvil: los Cuadros A1 y A3 replican el Cuadro 1 para ventanas de 8 y 12 años, respectivamente, mientras que los Cuadros A2 y A4 hacen lo propio con el Cuadro 2, también con ventanas de 8 y 12 años. 
Cuadro 2: Regresiones de panel con efectos fijos sobre la prociclicidad del gasto público con ventana móvil de 10 años

Prociclicidad del gasto público

(1)

\begin{tabular}{|c|c|c|c|c|}
\hline & $(1)$ & $(2)$ & $(3)$ & $(4)$ \\
\hline Tiempo con regla fiscal & $\begin{array}{c}0.133 \\
(0.124)\end{array}$ & & & \\
\hline Tiempo con SBR & & $\begin{array}{c}-0.360 * \\
(0.201)\end{array}$ & & $\begin{array}{c}-0.437^{* *} \\
(0.186)\end{array}$ \\
\hline Tiempo con BBR & & $\begin{array}{c}0.113 \\
(0.107)\end{array}$ & $\begin{array}{l}0.200^{*} \\
(0.101)\end{array}$ & \\
\hline Ratio deuda-PIB & $\begin{array}{c}0.017 \\
(0.110)\end{array}$ & $\begin{array}{c}0.008 \\
(0.111)\end{array}$ & $\begin{array}{c}0.015 \\
(0.112)\end{array}$ & $\begin{array}{c}0.002 \\
(0.114)\end{array}$ \\
\hline Crecimiento poblacional & $\begin{array}{c}-1.602 \\
(9.296)\end{array}$ & $\begin{array}{l}-0.156 \\
(9.388)\end{array}$ & $\begin{array}{l}-2.499 \\
(8.953)\end{array}$ & $\begin{array}{c}0.529 \\
(9.617)\end{array}$ \\
\hline Observaciones & 1455 & 1455 & 1455 & 1455 \\
\hline Número de países & 79 & 79 & 79 & 79 \\
\hline
\end{tabular}

Cabe destacar que estos resultados no implican en modo alguno causalidad, sino que se trata de un ejercicio exploratorio. Para poder establecer un vínculo causal, debería instrumentarse la implementación de reglas fiscales, en particular de la regla de balance estructural, ya que podría ser el caso que la aplicación de dicha regla fuera posible, justamente, en economías con una menor volatilidad del consumo. Una posible extensión de este trabajo, cuya contribución se encuentra en la discusión y las implicancias teóricas, sería explorar la cadena de causalidad en la elección de reglas fiscales y la volatilidad del consumo.

\section{Conclusiones}

El incremento en la implementación de reglas fiscales por parte de los países durante los últimos 30 años destaca la importancia de estudiar el efecto de las mismas sobre el desempeño de las economías. En ese sentido, la bibliografía señala que la adopción de reglas coincide con economías menos volátiles, menos procíclicas y menos proclives a caer en crisis de deuda. No obstante, no hay un consenso en la 
literatura sobre qué tipo de reglas son convenientes, teniendo como medida de desempeño el bienestar. Este trabajo lleva a un modelo teórico las reglas vinculadas con la sostenibilidad de la deuda: reglas de balance estructural, de presupuesto equilibrado y de límite a la deuda. Tratar las consecuencias de la implementación de dichas reglas en términos de bienestar en un marco común, permite hacer comparaciones. El trabajo presenta un modelo teórico en el cual se ponen a prueba los tres tipos de reglas. Las reglas de balance estructural maximizan el bienestar y son preferibles a las reglas de deuda y de presupuesto equilibrado, donde estas últimas además de distorsionar la suavización del consumo (disminuyendo así el bienestar), también maximizan la prociclicidad del gasto público. Estimaciones de datos en panel para una muestra global para el período 1985-2015 parecen sugerir que las reglas de balance estructural generan una disminución de la volatilidad del consumo privado significativa con respecto a los países que adoptan reglas de presupuesto equlibrado. Una posible extensión de este trabajo sería explorar la cadena de causalidad en la elección de reglas fiscales y la volatilidad del consumo.

\section{Referencias}

[1] Alesina, Alberto y Tamim Bayoumi. 1996. "The Costs and Benefits of Fiscal Rules: Evidence from U.S. States". Documento de trabajo 5614, NBER.

[2] Ardanáz, Martín, Eduardo Cavallo, Alejandro Izquierdo y Jorge Puig. 2019. "Growth friendly fiscal rules? Safeguarding public investment from budget cuts through fiscal rule design". Manuscrito no publicado, BID.

[3] Amador, Manuel, Iván Werning y George-Marios Angeletos. 2006. "Commitment vs. Flexibility". Econometrica, 74(2):365-396.

[4] Asatryan, Zareh, César Castellón y Thomas Stratmann. 2018. "Balanced budget rules and fiscal outcomes: Evidence from historical constitutions". Journal of Public Economics 167(2018):105-119.

[5] Azzimonti, Marina, Marco Battaglini y Stephen Coate. 2016. "The costs and benefits of balanced budget rules: Lessons from a political economy model of fiscal policy". Journal of Public Economics, 136(C):45-61. 
[6] Caselli, Francesca y Julien Reynaud. 2019. "Do Fiscal Rules Cause Better Fiscal Balances? A New Instrumental Variable Strategy". Documento de trabajo 19/49, FMI.

[7] Debrun, Xavier, Laurent Moulin, Alessandro Turrini, Joaquim Ayuso-i-Casals y Manmohan S. Kumar. 2008. "Tied to the mast? National fiscal rules in the European Union". Economic Policy, 23(54):298-362.

[8] Debrun, Xavier y Lars Jonung. 2018. "Under Threat: Rules-Based Fiscal Policy and How to Preserve It". Working Papers 2018:29, Lund University, Department of Economics.

[9] Drazen, Allan. (2013). "Fiscal Rules from a Political Economy Perspective". En Rules-Based Fiscal Policy in Emerging Markets, ed. G. Kopits, 15-29. London: Palgrave Macmillan.

[10] Eyraud, Luc, Xavier Debrun, Andrew Hodge, Victor Lledó y Catherine A. Pattillo. 2018. "Second-Generation Fiscal Rules; Balancing Simplicity, Flexibility, and Enforceability". Notas de discusión IMF 18/04, FMI.

[11] Fatas, Antonio e Ilian Mihov. 2004. "The Macroeconomic Effects of Fiscal Rules in the US States". Documento de discusión 4372, CEPR.

[12] Frankel, Jeffrey. 2011. "A Solution to Overoptimistic Forecasts and Fiscal Procyclicality: The Structural Budget Institutions Pioneered by Chile". Documento de trabajo 11-012, Harvard University, John F. Kennedy School of Government.

[13] Frankel, Jeffrey, Carlos Végh y Guillermo Vuletin. 2013. "On graduation from fiscal procyclicality". Journal of Development Economics, 100(1):32-47.

[14] Gatti, Nicolás y Jorge P. Puig. 2011. "Prociclicidad del gasto público en Argentina e implicancias para el diseño de políticas de federalismo fiscal". Asociación Argentina de Economía Política, XLVI Reunión Anual.

[15] Halac, Marina y Pierre Yared. 2014. "Fiscal Rules and Discretion Under Persistent Shocks". Econometrica, 82:1557-1614. 
[16] Hatchondo, Juan Carlos, Francisco Roch y Leonardo Martinez. 2012. "Fiscal Rules and the Sovereign Default Premium". Documento de trabajo 12/30, FMI.

[17] Izquierdo, Alejandro, Carola Pessino y Guillermo Vuletin. 2017. Mejor gasto para mejores vidas: Cómo América Latina y el Caribe pueden hacer más con menos. Washington D.C.: BID.

[18] Leeper, Eric. 1991. "Equilibria under 'active' and 'passive' monetary and fiscal policies". Journal of Monetary Economics, 27:129-147.

[19] Lledó, Victor, Sungwook Yoon, Xiangming Fang, Samba Mbaye y Young Kim (2017). "Fiscal Rules at a Glance"Background paper, FMI.

[20] Lucas, Robert y Nancy Stokey. 1983. "Optimal Fiscal and Monetary Policy in an Economy without capital". Journal of Monetary Economics, 12:55-93.

[21] Marcel, Mario. (2013). "The Structural Balance Rule in Chile: Ten Years, Ten Lessons". Documento de discusión 289, BID.

[22] Mendes, Arthur y Steven Pennings. 2017. "Consumption Smoothing and Shock Persistence: Optimal Simple Fiscal Rules for Commodity Exporters". Documento de trabajo sobre investigaciones relativas a políticas 8035, Banco Mundial.

[23] Milesi-Ferretti, Gian Maria. 2004. "Good, bad or ugly? On the effects of fiscal rules with creative accounting". Journal of Public Economics, 88(1-2):377-394.

[24] Owyang, Michael T., Valerie A. Ramey y Sarah Zubairy. 2013. "Are Government Spending Multipliers Greater During Periods of Slack? Evidence from 20th Century Historical Data”. Documento de trabajo 18769, NBER.

[25] Ramey, Garey y Valerie A. Ramey. 1995. "Cross-Country Evidence on the Link between Volatility and Growth". American Economic Review, 85(5):1138-1151.

[26] Riera-Crichton, Daniel, Carlos A. Végh y Guillermo Vuletin. 2015. "Procyclical and countercyclical fiscal multipliers: Evidence from OECD countries". Journal of International Money and Finance, 52(C): 15-31. 
[27] Schimtt-Grohé, Stephanie, y Martín Uribe. 2006. "Optimal simple and implementable monetary and fiscal rules". Journal of Monetary Economics, 54: 17021725.

[28] Sorensen, Bent, Lisa Wu y Oved Yosha. 2001. "Output fluctuations and fiscal policy: U.S. state and local governments 1978-1994". European Economic Review 45: 1271-1310.

[29] Sturzenegger, Federico y Rogério Werneck. 2006. "Fiscal Federalism and Procyclical Spending: The Cases of Argentina and Brazil". Económica, 52(1-2).

[30] Végh, Carlos A. 2013. Open Economy Macroeconomics in Developing Countries. Boston: M.I.T. Press.

[31] Wyplosz, Charles. 2012. "Fiscal Rules: Theoretical Issues and Historical Experiences". Documento de trabajo 17884, NBER.

\section{Apéndice}

Los países incluidos en las estimaciones empíricas incluyen: Antigua y Barbuda, Alemania, Argentina, Armenia, Australia, Austria, Benín, Bélgica, Botsuana, Brasil, Bulgaria, Burkina Faso, Burundi, Cabo Verde, Camerún, Canadá, República Centroafricana, República Checa, Chad, Chile, Chipre, Colombia, República de Congo, Costa Rica, Dinamarca, Dominica, Ecuador, Eslovaquia, España, Estados Unidos, Estonia, Finlandia, Francia, Georgia, Granada, Grecia, Guinea-Bisáu, Hong Kong, Hungría, India, Indonesia, Irán, Irlanda, Islandia, Israel, Italia, Jamaica, Japón, Kenia, Kosovo, Letonia, Liberia, Lituania, Malasia, Maldivas, Mauricio, Mexico, Mongolia, Montenegro, Namibia, Nueva Zelanda, Nigeria, Noruega, Nueva Zelanda, Países Bajos, Pakistán, Panamá, Paraguay, Perú, Polonia, Portugal, Reino Unido, Rumania, Rusia, Senegal, Serbia, Singapur, Sri Lanka, San Cristóbal y Nieves, Santa Lucía, Suecia, Suiza, Tanzania, Togo, Uganda y Uruguay. 
Figura A1: Correlación entre componentes cíclicos de ingresos tributarios y PIB real, 1980-2018

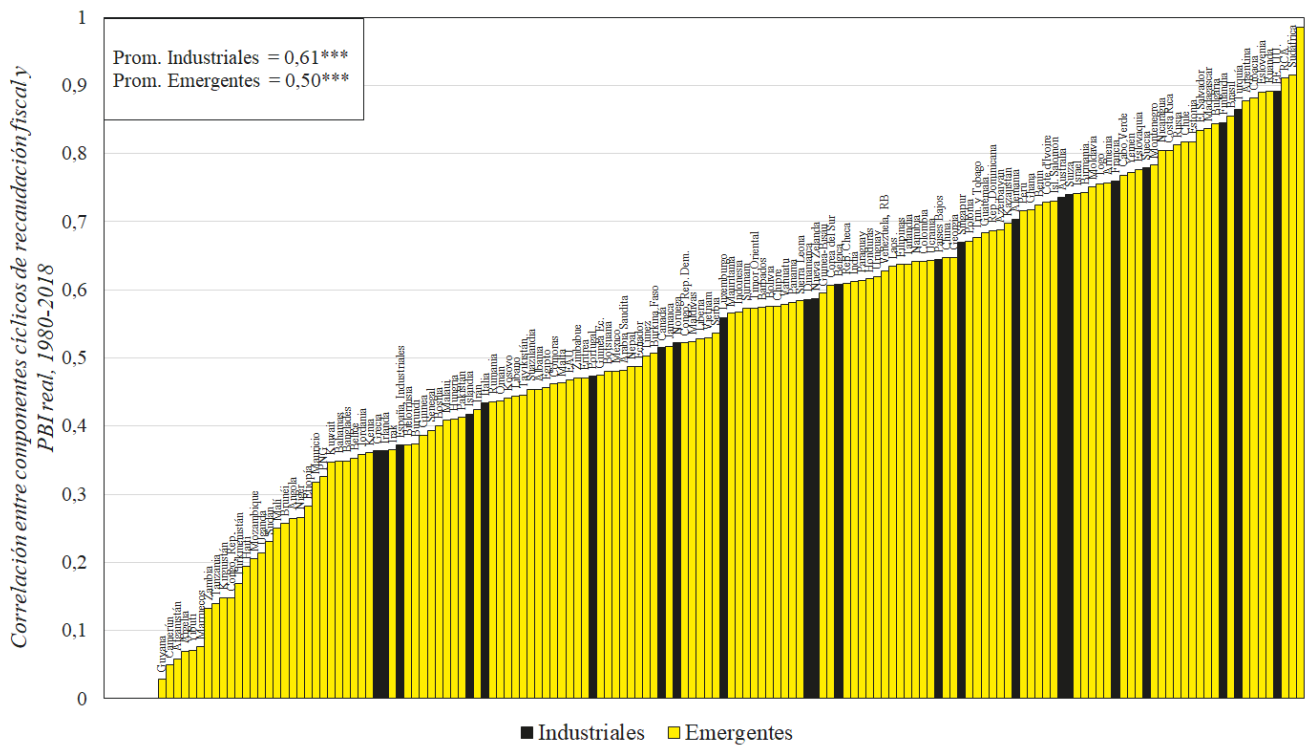

Fuente: elaboración propia basado en WEO (FMI).

Cuadro A1: Estadísticas descriptivas

Volatilidad del consumo privado Prociclicidad

$\begin{array}{lcc}\text { Muestra completa } & 0.053 & 0.032 \\ & (0.13) & (0.487) \\ \text { Regla fiscal } & 0.036 & 0.004 \\ & (0.142) & (0.456) \\ \text { BBR } & 0.037 & 0.032 \\ & (0.158) & (0.447) \\ \text { SBR } & 0.022 & -0.474 \\ & (0.034) & (0.383)\end{array}$

Nota: se presenta la mediana para cada muestra y el desvío estándar entre paréntesis. 
Cuadro A2: Regresiones de panel con efectos fijos sobre la volatilidad del consumo privado con ventana móvil de 8 años

\begin{tabular}{lcccc}
\hline & \multicolumn{4}{c}{ Volatilidad del consumo privado } \\
& $(1)$ & $(2)$ & $(3)$ & $(4)$ \\
\hline Tiempo con regla fiscal & -0.012 & & & \\
Tiempo con SBR & $(0.008)$ & & & \\
Tiempo con BBR & & $-0.015^{*}$ & & $-0.011^{* *}$ \\
& & $(0.008)$ & & $(0.005)$ \\
Ratio deuda-PIB & & -0.006 & -0.002 & \\
& -0.008 & -0.007 & -0.007 & -0.007 \\
Crecimiento poblacional & $(0.018)$ & $(0.018)$ & $(0.018)$ & $(0.019)$ \\
& 0.576 & 0.683 & 0.598 & 0.663 \\
Observaciones & $(0.578)$ & $(0.577)$ & $(0.576)$ & $(0.573)$ \\
Número de países & & & & \\
& 1554 & 1554 & 1554 & 1554 \\
& 78 & 78 & 78 & 78 \\
\hline
\end{tabular}

*** $p<0,01, * * p<0,05, * p<0,1$

Nota: Errores estándar robustos Eicker-Huber-White que corrigen por heterocedasticidad, entre paréntesis.

Cuadro A3: Regresiones de panel con efectos fijos sobre la prociclicidad del gasto público con ventana móvil de 8 años

Prociclicidad del gasto público

(1)

$(2)$

(3)

(4)

Tiempo con regla fiscal

0.114

$(0.116)$

Tiempo con SBR

$-0.373^{*}$

$-0.449 * *$

(0.200)

(0.187)

Tiempo con BBR

$0.1120 .193^{* *}$

$(0.096) \quad(0.093)$

Ratio deuda-PIB

$\begin{array}{lll}0.039 & 0.033 & 0.037\end{array}$

0.028

$\begin{array}{lll}(0.099) & (0.101) & (0.100)\end{array}$

(0.104)

Crecimiento poblacional

$-4.951 \quad-3.384$

$-5.777$

$-2.637$

(8.840)

$(8.927)$

(8.563)

(9.073)

Observaciones

$1498 \quad 1498 \quad 1498$

1498

Número de países

79

79

79

79

$* * * p<0,01, * * p<0,05, * p<0,1$

Nota: Errores estándar robustos Eicker-Huber-White que corrigen por heterocedasticidad, entre paréntesis. 
Cuadro A4: Regresiones de panel con efectos fijos sobre la volatilidad del consumo privado con ventana móvil de 12 años

\begin{tabular}{lcccc}
\hline & \multicolumn{3}{c}{ Volatilidad del consumo privado } \\
& $(1)$ & $(2)$ & $(3)$ & $(4)$ \\
\hline Tiempo con regla fiscal & -0.004 & & & \\
Tiempo con SBR & $(0.009)$ & & & \\
& & -0.012 & & $-0.015^{* *}$ \\
Tiempo con BBR & & $(0.008)$ & & $(0.006)$ \\
& & 0.003 & 0.007 & \\
Ratio deuda-PIB & 0.001 & 0.002 & 0.002 & 0.002 \\
& $(0.011)$ & $(0.011)$ & $(0.011)$ & $(0.012)$ \\
Crecimiento poblacional & 0.811 & $0.913^{*}$ & 0.840 & $0.921^{*}$ \\
& $(0.526)$ & $(0.526)$ & $(0.525)$ & $(0.525)$ \\
Observaciones & & & & \\
Número de países & 1430 & 1430 & 1430 & 1430 \\
\hline
\end{tabular}

$* * * p<0,01, * * p<0,05, * p<0,1$

Nota: Errores estándar robustos Eicker-Huber-White que corrigen por heterocedasticidad, entre paréntesis.

Cuadro A5: Regresiones de panel con efectos fijos sobre la prociclicidad del gasto público con ventana móvil de 12 años

Prociclicidad del gasto público

(1)

(2)

(3)

Tiempo con regla fiscal $\quad 0.149$

$(0.133)$

Tiempo con SBR

$-0.340$

$-0.422^{* *}$

$(0.206)$

$(0.185)$

Tiempo con BBR

$0.119 \quad 0.212^{*}$

$(0.122) \quad(0.111)$

Ratio deuda-PIB

0.034

$0.026 \quad 0.033$

0.020

(0.126)

(0.126)

$(0.129)$

(0.127)

Crecimiento poblacional

$-0.937$

0.305

$-1.745$

0.930

(10.141)

(10.236)

(9.818)

(10.447)

Observaciones

1408

1408

1408

1408

Número de países

79

79

79

79

*** $p<0,01, * * p<0,05,{ }^{*} p<0,1$

Nota: Errores estándar robustos Eicker-Huber-White que corrigen por heterocedasticidad, entre paréntesis. 